\title{
Simultaneous determination of multi-mycotoxins in palm kernel cake (PKC) using liquid chromatography-tandem mass spectrometry (LC-MS/MS)
}

\begin{abstract}
Palm kernel cake (PKC) is a useful source of protein and energy for livestock. Recently, it has been used as an ingredient in poultry feed. Mycotoxin contamination of PKC due to inappropriate handling during production and storage has increased public concern about economic losses and health risks for poultry and humans. This concern has accentuated the need for the evaluation of mycotoxins in PKC. Furthermore, a method for quantifying mycotoxins in PKC has so far not been established. The aims of this study were therefore (1) to develop a method for the simultaneous determination of mycotoxins in PKC and (2) to validate and verify the method. A liquid chromatography-tandem mass spectrometry (LCMS/MS) method using an electrospray ionisation interface (ESI) in both positive- and negative-ion modes was developed for the simultaneous determination of aflatoxins (AFB1, AFB2, AFG1 and AFG2), ochratoxin A (OTA), zearalenone (ZEA), deoxynivalenol (DON), fumonisins (FB1 and FB2), T-2 and HT-2 toxin in PKC. An optimum method using a $0.2 \mathrm{ml}$ min-1 flow rate, $0.2 \%$ formic acid in aqueous phase, $10 \%$ organic phase at the beginning and $90 \%$ organic phase at the end of the gradient was achieved. The extraction of mycotoxins was performed using a solvent mixture of acetonitrile-water-formic acid (79:20:1, v/v) without further clean-up. The mean recoveries of mycotoxins in spiked PKC samples ranged from $81 \%$ to $112 \%$. Limits of detection (LODs) and limits of quantification (LOQs) for mycotoxin standards and PKC samples ranged from 0.02 to $17.5 \mu \mathrm{g} \mathrm{kg}-1$ and from 0.06 to $58.0 \mu \mathrm{g} \mathrm{kg}-1$, respectively. Finally, the newly developed method was successfully applied to PKC samples. The results illustrated the fact that the method is efficient and accurate for the simultaneous multi-mycotoxin determination in $\mathrm{PKC}$, which can be ideal for routine analysis.
\end{abstract}

Keyword: LC-MS/MS; Multi-mycotoxins; Palm kernel cake; Optimisation; Simultaneous determination 